\title{
Ein Bibliothekar aus Wolfenbüttel!
}

Man hätte es sich eigentlich denken können. Indes, als die Sächsische Landesbibliothek - Staats- und Universitätsbibliothek Dresden vor zwei Jahrzehnten einen Stellvertretenden Generaldirektor suchte, da stand anderes vor Augen als nun gerade die frühneuzeitliche Buch- und Bibliotheksgeschichte: das Bauen selbstverständlich, sodann die tatsächliche strukturelle Integration der Vorgängereinrichtungen, nicht zuletzt die rapide wachsenden kapazitären wie qualitativen Anforderungen zumal auf der universitätsbibliothekarischen Seite.

Fünf Jahre später dann hatte sich die Lage merklich verändert. Das schöne Haus war bezogen. Und längst hatte es auch seine praktische Bewährung im Alltag bestanden. Die Bibliothek hatte sozusagen ihre Reiseflughöhe erreicht. Und das machte den Blick weiter - auch, als dem Haus ein neuer Generaldirektor zu bestellen war. Dabei wollten wir, was heutzutage in sprödestem Deutsch auch Informationsinfrastruktur genannt wird, durchaus emphatisch verstehen. Und dies hieß, und heißt es immer noch, dass noch andere Ziele anzusteuern waren - doch diese freilich auch - als diejenigen eingeführter bibliothekarischer Dienstleistungen.

Auf den digitalen Weltenwandel durfte die Bibliothek nicht bloß atemlos reagieren. Seine enorme, ebenso chancenreiche wie riskante Komplexität reflexiv begreifend, sollte sie vielmehr mitzugestalten versuchen, wohin die Reise geht. Die Dynamik studentischer und wissenschaftlicher Arbeits- und Kommunikationsformen würde sie weiterhin wahrnehmungsgenau begleiten und ihr Raum geben müssen; einschneidend verändern ja ihre Sozialfunktionen die Bibliothek zu einem akademischen Zentrum, in dem Anwesenheit ungleich wichtiger ist, als die Lobredner vollständiger Virtualisierung sich das vorstellen mögen. Und in eins damit gewinnt die kulturelle Ausstrahlung der Gedächtnisinstitution an Bedeutung: als Museum, als intellektueller Vermittlungsort, als besonderes Forum wacher gesellschaftlicher Selbstverständigung.

Thomas Bürger hat all dies im Blick, im Sinn, in der Hand. In den Jahren seiner Leitung erfüllen und entfalten sich in Dresden anspruchsvolle Konzepte dessen, was Bibliothek heute und in Zukunft sein könne. Seinem Rat und Urteil gibt das auch in Wissenschaftspolitik und wissenschaftlicher Selbstverwaltung besonderes Gewicht, nicht zuletzt bei der Deutschen Forschungsgemeinschaft. Es ist das Gewicht von praktischer Überzeugungskunst, von sensibler Tatkraft und diplomatischer Intellektualität. Und es verbindet sich mit dem optimistischen Charme dieses Bibliothekars, dem sehr viele zu Dank verpflichtet sind da-

๖ Open Access. (C) 2018 Peter Strohschneider, publiziert von De Gruyter. (@) BY-NC-ND Dieses Werk ist lizenziert unter der Creative Commons Attribution-NonCommercial-NoDerivatives 4.0 Lizenz. 
$24-$ Grußadressen

für, dass er sein Haus stets von denjenigen her denkt und gestaltet, für die es da ist, die Wissenschaftsgesellschaft und ihre Bürger.

Prof. Dr. Peter Strohschneider

Präsident der Deutschen Forschungsgemeinschaft 